\title{
Distributive Lattices and Hurewicz families
}

\author{
MARION SCHEEPERS
}

\begin{abstract}
Hurewicz and Rothberger respectively introduced prototypes of the selection properties $\mathrm{S}_{f i n}(\mathcal{A}, \mathcal{B})$ and $\mathrm{S}_{1}(\mathcal{A}, \mathcal{B})$. In the series of papers titled "Combinatorics of open covers" (see the bibliography) we learned that for various topologically significant families $\mathcal{A}$ and $\mathcal{B}$ these selection properties are intimately related to game theory and Ramsey theory. The similarity in techniques used there to explore these relationships suggests that there should be a general, unified way to obtain these results. In this paper we pursue one possibility by considering the selection principle $\mathrm{S}_{\text {fin }}(\mathcal{A}, \mathcal{B})$ for distributive lattices. The selection principle $S_{1}(\mathcal{A}, \mathcal{B})$ for distributive lattices will be treated in [3]. We use two examples throughout to illustrate the generality of the methods developed here.
\end{abstract}

\section{BASIC NOTIONS}

Fix a distributive lattice $\mathbb{L}$. Except when explicitly stated otherwise, our lattices are not assumed to be complemented, nor to have a largest or a least element, nor to have any completeness properties beyond those implied by the basic definition of a distributive lattice.

Let families $\mathcal{A}$ and $\mathcal{B}$ of subsets of the lattice $\mathbb{L}$ be given. The selection principle $\mathrm{S}_{\text {fin }}(\mathcal{A}, \mathcal{B})$ states that for each sequence $\left(O_{n}: n \in \mathbb{N}\right)$ from $\mathcal{A}$ there is a sequence $\left(T_{n}: n \in \mathbb{N}\right)$ such that $T_{n}$ is for each $n$ a finite subset of $O_{n}$, and $\cup_{n \in \mathbb{N}} T_{n}$ is an element of $\mathcal{B}$. It is evident from the definition that $S_{f i n}(\cdot, \cdot)$ is antimonotonic in the first variable, and monotonic in the second variable: More precisely, let $\mathcal{A}, \mathcal{B}$, $\mathcal{C}$ and $\mathcal{D}$ be families of subsets of $\mathbb{L}$ such that $\mathcal{A} \subseteq \mathcal{C}$ and $\mathcal{B} \subseteq \mathcal{D}$. Then we have the implications

$$
\mathrm{S}_{\text {fin }}(\mathcal{C}, \mathcal{B}) \Rightarrow \mathrm{S}_{\text {fin }}(\mathcal{A}, \mathcal{B})
$$

and

$$
\mathrm{S}_{\text {fin }}(\mathcal{A}, \mathcal{B}) \Rightarrow \mathrm{S}_{\text {fin }}(\mathcal{A}, \mathcal{D})
$$

We denote these interrelationships by a diagram as below, where the property at the initial point of an arrow implies the property at the arrow's terminal point:

2000 Mathematics Subject Classification. Primary: 03E05, 05D10, 06B99, 91A44.

Key words and phrases. game, selection principle, lattice, $\mathcal{A}$-tree, Hurewicz $\mathcal{A}$-tree, Hurewicz pairs, Ramsey family, partition relation, selectable pair. 


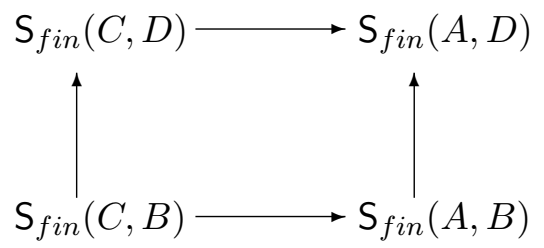

There is a natural game, $\mathrm{G}_{f i n}(\mathcal{A}, \mathcal{B})$, associated with this selection principle: Players ONE and TWO play an inning per positive integer. In the $n$-th inning ONE first chooses a set $O_{n} \in \mathcal{A}$; then TWO responds by choosing a finite subset $T_{n}$ of $O_{n}$. TWO wins a play $O_{1}, T_{1}, \ldots, O_{n}, T_{n}, \ldots$ if $\cup_{n \in \mathbb{N}} T_{n} \in \mathcal{B}$; otherwise, ONE wins.

If ONE has no winning strategy in the game $\mathrm{G}_{\text {fin }}(\mathcal{A}, \mathcal{B})$, then the selection principle $\mathrm{S}_{\text {fin }}(\mathcal{A}, \mathcal{B})$ holds. The converse implication is not always true. When it is in fact true, the game is a powerful tool to analyse the combinatorial properties of the families $\mathcal{A}$ and $\mathcal{B}$. In this paper we identify circumstances under which this converse is true. The concepts of an $\mathcal{A}$-tree and of Hurewicz pairs are central to this. In Sections 2 we develop the notion of a Hurewicz $\mathcal{A}$-tree, and prove the Fundamental Theorem of Hurewicz $\mathcal{A}$-trees (Theorem 2) from which much of the theory of $\mathrm{S}_{\text {fin }}(\mathcal{A}, \mathcal{B})$ can be derived. To derive game-theoretic results from Theorem 2 the pair $(\mathcal{A}, \mathcal{B})$ should have properties permitting various constructions in $\mathbb{L}$. The notion of a Hurewicz pair, introduced in Section 3, is intended to capture these requirements. We derive the Fundamental Theorem for Hurewicz Pairs (Theorem 7): If $(\mathcal{A}, \mathcal{B})$ is a Hurewicz pair, then $\mathrm{S}_{\text {fin }}(\mathcal{A}, \mathcal{B})$ holds if, and only if, ONE has no winning strategy in $\mathrm{G}_{f i n}(\mathcal{A}, \mathcal{B})$.

Next we take up the connections of $\mathrm{S}_{\text {fin }}(\mathcal{A}, \mathcal{B})$ with Ramsey theory. For $k$ a positive integer the symbol $\mathcal{A} \rightarrow\lceil\mathcal{B}\rceil_{k}^{2}$ denotes that for each $A \in \mathcal{A}$ and for each function $f:[A]^{2} \rightarrow\{1, \ldots, k\}$ there is a set $B \subset A$, an $i \in\{1, \ldots, k\}$, and a partition $B=\cup_{n \in \mathbb{N}} B_{n}$ of $B$ into pairwise disjoint finite sets, such that for all $\{a, b\} \in[B]^{2}$ with for each $n\left|\{a, b\} \cap B_{n}\right| \leq 1$, we have $f(\{a, b\})=i$. This is an example of a partition relation. We shall call it the Baumgartner-Taylor partition relation since a version of it was introduced by these mathematicians in [4]. They introduced it to give a Ramsey-theoretic characterization of $P$-point ultrafilters. In Sections 4 and 5 we identify circumstances under which truth of the partition relation is equivalent to truth of the selection principle $\mathrm{S}_{\text {fin }}(\mathcal{A}, \mathcal{B})$. The concepts of a Ramsey family and of a selectable pair are central to this task. In Section 4 we prove the Fundamental Theorem of Ramsey Families (Theorem 12), stating that for certain Ramsey families $\mathcal{A}$, if ONE has no winning strategy in the game $\mathrm{G}_{\text {fin }}(\mathcal{A}, \mathcal{B})$, then $\mathcal{A} \rightarrow\lceil\mathcal{B}\rceil_{k}^{2}$ holds.

In Section 5 we prove the Fundamental Theorem of Selectable Pairs (Theorem 17), stating that under appropriate hypotheses, if $\mathcal{A} \rightarrow\lceil\mathcal{B}\rceil_{k}^{2}$ holds, then $\mathrm{S}_{\text {fin }}(\mathcal{A}, \mathcal{B})$ holds. 
For each of the three situations $\left(S_{\text {fin }}(\mathcal{A}, \mathcal{B})\right.$, the game $\mathrm{G}_{\text {fin }}(\mathcal{A}, \mathcal{B})$ or partition relation $\mathcal{A} \rightarrow\lceil\mathcal{B}\rceil_{k}^{2}$ ), the only members of $\mathcal{B}$ that matter are those which are subsets of $\cup \mathcal{A}$. Thus, we will make the further assumption throughout this paper about $\mathcal{A}$ and $\mathcal{B}$ :

\section{Hypothesis 1. $\cup \mathcal{B} \subset \cup \mathcal{A}$.}

Various well-known concepts are important in the study of selection principles, and are now introduced. For elements $a$ and $b$ of lattice $\mathbb{L}$ define $a \leq b$ if $b=a \vee b$. We write $a<b$ to denote that $a \leq b$ and $a \neq b$. Then $<$ defines a partial ordering on the set $\mathbb{L}$. For subsets $A$ and $B$ of $\mathbb{L}$ we define:

Definition 1. (1) A refines $B$, written $A \prec B$, if there is for each $a \in A$ a $b \in B$ such that $a \leq b$. If $A \prec B$ and $B \subset A, B$ is cofinal in $A$.

(2) $\mathcal{A}$ is refinement closed if whenever $B \subset \cup \mathcal{A}$ is refined by an element of $\mathcal{A}$, then $B \in \mathcal{A}$. $\mathcal{A}$ is cofinally closed if every cofinal subset of an element of $\mathcal{A}$ is also an element of $\mathcal{A}$.

In the definition of " $A$ refines $B$ " we do not require that $A$ be a subset of $B$.

\section{Hurewicz $\mathcal{A}$-Trees}

Definition 2. (1) A family $\left(T_{\tau}: \tau \in{ }^{<\omega} \mathbb{N}\right)$ of elements of $\mathbb{L}$ is said to be a $\mathcal{A}$-tree if:

For each $\tau \in<\omega \mathbb{N}\left\{T_{\tau \frown n}: n \in \mathbb{N}\right\} \in \mathcal{A}$.

(2) For $f$ in ${ }^{\omega} \mathbb{N}$ the subset $\left\{T_{f\left\lceil_{n}\right.}: n \in \mathbb{N}\right\}$ of an $\mathcal{A}$-tree is said to be a branch. It is said to be a $\mathcal{B}$-branch if $\left\{T_{f\left\lceil_{n}\right.}: n \in \mathbb{N}\right\}$ is a member of $\mathcal{B}$.

(3) An $\mathcal{A}$-tree $\left(T_{\tau}: \tau \in<\omega \mathbb{N}\right)$ is said to be a Hurewicz $\mathcal{A}$-tree if:

HT1 For each $\tau$ if $m<n$ then $T_{\tau \frown m} \leq T_{\tau \frown n}$;

HT2 For each $\tau$ and for each $n, T_{\tau} \leq T_{\tau \frown n}$.

Several basic constructions play an important role in determining when an $\mathcal{A}$ tree has a $\mathcal{B}$-branch.

Definition 3. Let $\left(T_{\tau}: \tau \in<\omega \mathbb{N}\right)$ be an $\mathcal{A}$-tree.

(1) For each $n$ and for each $k$ in $\mathbb{N}$ put

$$
C_{k}^{n}= \begin{cases}T_{k}, & \text { if } n=1 \\ \left(\wedge\left\{C_{k}^{n-1} \wedge T_{\tau \frown k}: \tau \in{ }^{n-1} \mathbb{N}\right\}\right), & \text { otherwise. }\end{cases}
$$

(2) For each $n$ in $\mathbb{N}$ put $\mathcal{U}_{n}=\left\{C_{k}^{n}: k \in \mathbb{N}\right\}$. The sequence $\left(\mathcal{U}_{n}: n \in \mathbb{N}\right)$ is said to be the linearization of the $\mathcal{A}$-tree.

The definition of $C_{k}^{n}$ for $n>1$ may appear to require that the lattice in question has a certain degree of completeness under infinitary operations. This is only appearances, since only finitely many terms in the definition have an effect on computing $C_{k}^{n}$ :

Lemma 1. Let $\left(T_{\tau}: \tau \in<\omega \mathbb{N}\right)$ be a Hurewicz $\mathcal{A}$-tree. 
(1) For each $n$ and for each $\left(i_{1}, \ldots, i_{n}\right)$ such that $k \leq \max \left\{i_{1}, \ldots, i_{n}\right\}$ we have

$$
C_{k}^{n} \leq T_{\left(i_{1}, \ldots, i_{n}\right)}
$$

(2) For each $n$ : If $k<\ell$, then $C_{k}^{n} \leq C_{\ell}^{n}$.

Proof. The proof of 1 is by induction on $n$.

$\underline{n=1}$ : If $k \leq i_{1}$ then by HT1, $T_{k} \leq T_{i_{1}}$, i.e., $C_{k}^{1} \leq T_{i_{1}}$.

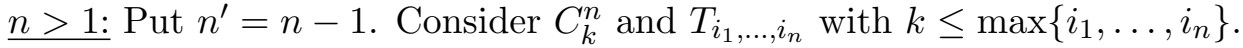

Case 1: $i_{n}<k$.

Then $k \leq \max \left\{i_{1}, \ldots, i_{n^{\prime}}\right\}$ and so by HT2

$$
C_{k}^{n^{\prime}} \leq T_{i_{1}, \ldots, i_{n^{\prime}}} \leq T_{i_{1}, \ldots, i_{n^{\prime}}, i_{n}} .
$$

But since $C_{k}^{n} \leq C_{k}^{n^{\prime}}$ we are done.

Case 2: $k \leq i_{n}$.

Then by definition and by HT1, $C_{k}^{n} \leq T_{i_{1}, \ldots, i_{n^{\prime}}, k} \leq T_{i_{1}, \ldots, i_{n^{\prime}}, i_{n}}$ and we are done. Also the proof of 2 is by induction on $n$.

Case 1: $n=1$. Then $C_{k}^{n}=T_{k}$ and $C_{\ell}^{n}=T_{\ell}$. Since these are members of a Hurewics $\mathcal{A}$-tree we have from HT1 that $C_{k}^{n} \leq C_{\ell}^{n}$. HT1,

Case 2: $n>1$ and the result is true below $n$. Thus, $C_{k}^{n-1} \leq C_{\ell}^{n-1}$. Also, by

$$
\wedge\left\{T_{\sigma \frown k}: \sigma \in{ }^{n} \mathbb{N}\right\} \leq \wedge\left\{T_{\sigma \frown \ell}: \sigma \in{ }^{n} \mathbb{N}\right\} .
$$

The result follows.

Much of the theory of the selection principle $\mathrm{S}_{\text {fin }}(\mathcal{A}, \mathcal{B})$ can be derived from the following fact:

Theorem 2 (Fundamental Theorem for Hurewicz $\mathcal{A}$-trees). Assume that $(\mathcal{A}, \mathcal{B})$ has the following properties:

(1) Each term of the linearization of each Hurewicz $\mathcal{A}$-tree is a member of $\mathcal{A}$,

(2) $\mathcal{B}$ is refinement closed (and thus cofinally closed) If $\mathrm{S}_{\text {fin }}(\mathcal{A}, \mathcal{B})$ holds, then each Hurewicz $\mathcal{A}$-tree has a $\mathcal{B}$-branch.

Proof. Let $\left(T_{\tau}: \tau \in{ }^{<\omega} \mathbb{N}\right)$ be a Hurewicz $\mathcal{A}$-tree. Let $\left(U_{n}: n \in \mathbb{N}\right)$ be its linearization. Each $U_{n}$ is a member of $\mathcal{A}$. Apply $\mathrm{S}_{f i n}(\mathcal{A}, \mathcal{B})$ to the sequence $\left(U_{n}: n \in \mathbb{N}\right)$ and choose for each $n$ a finite set $F_{n} \subset U_{n}$ such that $\cup_{n \in \mathbb{N}} F_{n}$ is a member of $\mathcal{B}$. For each $n$ choose a finite subset $G_{n}$ of $U_{n}$ such that $F_{n} \subset G_{n}$, and with $k_{n}$ maximal with $C_{k_{n}}^{n} \in G_{n}$ we have for $j<\ell$ that $k_{j}<k_{\ell}$. Then by 3 also $\cup_{n \in \mathbb{N}} G_{n}$ is a member of $\mathcal{B}$.

By Lemma 1 part 2, each element of $G_{n}$ is $\leq C_{k_{n}}^{n}$. Thus, $\left\{C_{k_{n}}^{n}: n \in \mathbb{N}\right\}$ is cofinal in $\cup_{n \in \mathbb{N}} G_{n}$, and so by 2 is also a member of $\mathcal{B}$. Since $k_{1}<k_{2}<\cdots<k_{n}<\ldots$ we have from Lemma 1 part 1 that for each $n$ also

$$
C_{k_{n}}^{n} \leq T_{k_{1}, \ldots, k_{n}} .
$$

Again applying 3 we see that $\left\{T_{k_{1}, \ldots, k_{n}}: n \in \mathbb{N}\right\}$ is a member of $\mathcal{B}$. But this set is a $\mathcal{B}$-branch of the Hurewicz $\mathcal{A}$-tree. 
3. Hurewicz pairs and the Game $\mathrm{G}_{f i n}(\mathcal{A}, \mathcal{B}): \operatorname{From}_{\text {fin }}(\mathcal{A}, \mathcal{B})$ to

$$
\mathrm{G}_{\text {fin }}(\mathcal{A}, \mathcal{B}) \text {. }
$$

Game-theoretic applications of the fundamental theorem of Hurewicz $\mathcal{A}$-trees depend on being able to construct from strategies of player ONE such trees whose linearizations have appropriate properties. The notion of a Hurewicz pair, which we now define, captures some of the requirements for such constructions. $\mathcal{A}$ is said to be Lindelöf if each element of $\mathcal{A}$ has a countable subset which is in $\mathcal{A}$. It is evident that $\mathrm{S}_{\text {fin }}(\mathcal{A}, \mathcal{A})$ implies that $\mathcal{A}$ is Lindelöf; the converse need not be true.

Definition 4. $(\mathcal{A}, \mathcal{B})$ is a Hurewicz pair if it has the following properties:

$\mathrm{H} 1 \mathcal{A}$ is Lindelöf;

H2 For each $A$ in $\mathcal{A},\{\wedge \mathcal{F}: \emptyset \neq \mathcal{F} \subset A$ finite $\}$ is an element of $\mathcal{A}$;

$\mathrm{H} 3 \mathcal{A}$ is refinement closed;

H4 For each nonempty finite set $F \subset \cup \mathcal{A}, \vee F$ is in $\cup \mathcal{A}$.

H5 Each term of the linearization of a Hurewicz $\mathcal{A}$-tree is a member of $\mathcal{A}$.

H6 For each $B \in \mathcal{B}$ and each $C \subset \cup \mathcal{A}$, if $B \subset C$, then $C \in \mathcal{B}$

H7 For each $B \subset \cup \mathcal{A}$, if $\{\vee \mathcal{F}: \emptyset \neq \mathcal{F} \subset B$ finite $\} \in \mathcal{B}$, then $B \in \mathcal{B}$.

$\mathrm{H} 8 \mathcal{B}$ is refinement closed

$\mathcal{A}$ is a Hurewicz family if $(\mathcal{A}, \mathcal{A})$ is a Hurewicz pair.

Definition 5. For a given family $\mathcal{A}$ let $\mathcal{A}_{\Omega}$ denote the set of $A \in \mathcal{A}$ which have the following property:

For each $k$ and each partition $A=A_{1} \cup \cdots \cup A_{k}$ of $A$ there is a $j \leq k$ with $A_{j} \in \mathcal{A}$.

Lemma 3. Let $(\mathcal{A}, \mathcal{B})$ be a Hurewicz pair.

(1) For each $A \in \mathcal{A}$, for each nonempty finite subset $F$ of $A, \wedge F \in \cup \mathcal{A}$.

(2) For $A \in \mathcal{A}$ and $b \in \cup \mathcal{A},\{a \vee b: a \in A\} \in \mathcal{A}$.

(3) For each $A \in \mathcal{A}$ the set $A^{*}:=\{\vee F: \emptyset \neq F \subset A$ finite $\}$ is in $\mathcal{A}_{\Omega}$.

(4) Each $A \in \mathcal{A}$ contains a countable subset $B$ such that whenever $a, b \in B$, then either $a<b$ or $b<a$, and every infinite subset of $B$ is in $\mathcal{A}$.

(5) If $\mathcal{F}$ is a family of elements of $\mathcal{A}$, then $\cup \mathcal{F}$ is an element of $\mathcal{A}$.

Proof. 1 follows immediately from [H2].

2 follows from [H3] and [H4].

3 follows from [H3] and [H4] that $A^{*}$ is in $\mathcal{A}$. To see that $A^{*}$ is in $\mathcal{A}_{\Omega}$, choose a partition $A^{*}=B_{1} \cup \cdots \cup B_{k}$. Then $A$ refines some $B_{j}$ (For suppose the contrary and choose for each $j$ an $a_{j} \in A$ but there is no $b \in B_{j}$ with $a_{j} \leq b$. Then put $c=\vee_{j \leq k} a_{j}$. This is an element of $A^{*}$, so a member of some $B_{j}$. But then $a_{j} \leq c$, contradicting the choice of $a_{j}$ ). By [H3], $B_{j}$ is in $\mathcal{A}$.

4 By [H1], let $\left\{a_{n}: n=1,2,3, \ldots\right\}$ be a subset of $A$ which is in $\mathcal{A}$. For each $n$ put $b_{n}=\vee_{j \leq n} a_{j}$. By [H4] each $b_{n}$ is an element of $\cup \mathcal{A}$. Put $B=\left\{b_{n}: n=1,2,3, \ldots\right\}$. Then as $A$ refines $B$, [H3] implies that $B \in \mathcal{A}$. $B$ is as required.

5 Each element of $\mathcal{F}$ refines $\cup \mathcal{F}$. Apply [H3]. 
We now consider for the families $\mathcal{A}$ and $\mathcal{B}$ of subsets of $\mathbb{L}$ the infinite game $\mathrm{G}_{\text {fin }}(\mathcal{A}, \mathcal{B})$ that was defined in the introduction. First we analyze properties of winning strategies for player ONE in this game. We shall call finite sequences $\left(F_{1}, \ldots, F_{n}\right)$ admissible if

(1) $j \leq n$ there is a $A_{j} \in \mathcal{A}$ such that $F_{j} \subseteq A_{j}$, and

(2) each $F_{j}$ is finite.

Lemma 4. Let $(\mathcal{A}, \mathcal{B})$ be a Hurewicz pair. If ONE has a winning strategy in the game $\mathrm{G}_{\text {fin }}(\mathcal{A}, \mathcal{B})$ then $O N E$ has a winning strategy $F$ such that for each admissible sequence $\left(F_{1}, \ldots, F_{n}\right)$, for each $b \in F\left(F_{1}, \ldots, F_{n}\right)$, we have $\vee_{j \leq n}\left(\vee F_{j}\right) \leq b$.

Proof. Let $\sigma$ be a winning strategy for $\mathrm{ONE}$ in $\mathrm{G}_{f i n}(\mathcal{A}, \mathcal{B})$. Let $\prec$ be a well-order of the set of finite subsets of $S$. Define a strategy $F$ for ONE as follows:

- $F(\emptyset)=\sigma(\emptyset)$

- For $F_{1} \subset F(\emptyset)$ finite, first compute $\sigma\left(F_{1}\right)$ and $b=\vee F_{1}$. By [H4] there is an $A \in \mathcal{A}$ with $b \in A$. Put

$$
F\left(F_{1}\right)=\left\{b \vee c: c \in \sigma\left(F_{1}\right)\right\}
$$

By 2 of Lemma $3, F\left(F_{1}\right) \in \mathcal{A}$.

- For $F_{2} \subset F\left(F_{1}\right)$ finite, let $W_{2} \subset \sigma\left(F_{1}\right)$ be the $\prec$-first finite set with $F_{2}=\left\{W \vee\left(\vee F_{1}\right): W \in W_{2}\right\}$. Compute $\sigma\left(F_{1}, W_{2}\right)$ and define $F\left(F_{1}, F_{2}\right)=$ $\left\{\left(\vee F_{2}\right) \vee c: c \in \sigma\left(F_{1}, W_{2}\right)\right\}$. By 1 of Lemma 3 and by $\mathrm{H} 4 F\left(F_{1}, F_{2}\right) \in \mathcal{A}$.

- For $F_{n+1} \subset F\left(F_{1}, \ldots, F_{n}\right)$ finite, for $2 \leq j \leq n$ let $W_{j+1} \subset \sigma\left(F_{1}, W_{2}, \ldots, W_{j}\right)$ be the $\prec$-first finite set with $F_{j+1}=\left\{W \vee\left(\vee_{i \leq j} \vee F_{i}\right): W \in W_{j+1}\right\}$. Compute $\sigma\left(F_{1}, W_{2}, \ldots, W_{n+1}\right)$ and define $F\left(F_{1}, F_{2}, \ldots, F_{n+1}\right)=\left\{\left(\vee F_{n+1}\right) \vee c\right.$ : $\left.c \in \sigma\left(F_{1}, W_{2}, \ldots, W_{n+1}\right)\right\}$. By 1 of Lemma 3 and by [H4] $F\left(F_{1}, F_{2}, \ldots, F_{n+1}\right) \in$ $\mathcal{A}$.

To see that $F$ is a winning strategy for ONE, consider an $F$-play

$$
F(\emptyset), T_{1}, F\left(T_{1}\right), T_{2}, F\left(T_{1}, T_{2}\right), \ldots
$$

From the definition of $F$ recursively choose finite sets $W_{1}, \ldots, W_{n}, \ldots$ such that

(1) $W_{1}=T_{1}$,

(2) $W_{n+1}$ is the $\prec$-least finite set with $W_{n+1} \subseteq \sigma\left(W_{1}, \ldots, W_{n}\right)$ and $T_{n+1}=$ $\left\{\left(\vee T_{n}\right) \vee b: b \in W_{n+1}\right\}$.

Then

$$
\sigma(\emptyset), W_{1}, \sigma\left(W_{1}\right), W_{2}, \sigma\left(W_{1}, W_{2}\right), \ldots
$$

is a $\sigma$-play of $\mathrm{G}_{f i n}(\mathcal{A}, \mathcal{B})$, so won by $\mathrm{ONE}$, so $\cup_{n \in \mathbb{N}} W_{n} \notin \mathcal{B}$. Then by the contrapositive of [H7] also $\left\{\vee F: \emptyset \neq F \subseteq \cup_{n \in \mathbb{N}} W_{n}\right.$ finite $\}$ is not in $\mathcal{B}$. Then by [H8] also $\cup_{n \in \mathbb{N}} T_{n}$ is not in $\mathcal{B}$. Thus ONE wins the $F$-play we are considering.

Lemma 5. Let $(\mathcal{A}, \mathcal{B})$ be a Hurewicz pair. If $\sigma$ is a winning strategy for ONE in $\mathrm{G}_{\text {fin }}(\mathcal{A}, \mathcal{B})$, then each strategy $\tau$ of $O N E$ which satisfies

$$
\tau\left(T_{1}, \ldots, T_{n}\right) \subseteq \sigma\left(T_{1}, \ldots, T_{n}\right),
$$

each admissible sequence $\left(T_{1}, \ldots, T_{n}\right)$ is a winning strategy. 
Proof. Consider a $\tau$-play

$$
\tau(\emptyset), T_{1}, \tau\left(T_{1}\right), T_{2}, \tau\left(T_{1}, T_{2}\right), \ldots
$$

We have $\tau(\emptyset) \subseteq \sigma(\emptyset)$, and so $T_{1} \subseteq \sigma(\emptyset)$. For each $n, T_{n+1} \subseteq \tau\left(T_{1}, \ldots, T_{n}\right) \subseteq$ $\sigma\left(T_{1}, \ldots, T_{n}\right)$. Thus

$$
\sigma(\emptyset), T_{1}, \sigma\left(T_{1}\right), T_{2}, \sigma\left(T_{1}, T_{2}\right), \ldots
$$

is a $\sigma$-play of $\mathrm{G}_{\text {fin }}(\mathcal{A}, \mathcal{B})$, and so won by ONE. This means $\cup_{n \in \mathbb{N}} T_{n} \notin \mathcal{B}$. Thus the original $\tau$-play is won by ONE.

Lemma 6. Let $(\mathcal{A}, \mathcal{B})$ be a Hurewicz pair. If ONE has a winning strategy in the game $\mathrm{G}_{\text {fin }}(\mathcal{A}, \mathcal{B})$, then $O N E$ has a winning strategy $F$ which calls in each inning on ONE to play a member of $\mathcal{A}$ which under the partial order $<$ is an $\omega$-sequence.

Proof. Let $\sigma$ be a winning strategy for ONE. By Lemma 5 and by [H1] we may assume that in each inning $\sigma$ calls on ONE to play a countable element of $\mathcal{A}$.

Define a new strategy $G$ for ONE as follows:

(1) With $\sigma(\emptyset)=\left\{b_{n}: n \in \mathbb{N}\right\}$ define $G(\emptyset)=\left\{\vee_{j \leq n} b_{j}: n \in \mathbb{N}\right\}$. By [H3] and the proof of 4 of Lemma $3, G(\emptyset) \in \mathcal{A}$.

(2) To define $G\left(T_{1}, \ldots, T_{n}\right)$, choose for each $j \leq n$ a minimal finite set $F_{j}$ with $F_{1} \subset \sigma(\emptyset)$ and the elements of $T_{1}$ sups of elements of $F_{1}$, and for $j>1, F_{j} \subset \sigma\left(F_{1}, \ldots, F_{j-1}\right)$ and the elements of $T_{j}$ sups of elements of $F_{j}$. Suppose that $\sigma\left(F_{1}, \ldots, F_{n}\right)=\left\{b_{m}: m \in \mathbb{N}\right\} \in \mathcal{A}$ and define

$$
G\left(T_{1}, \ldots, T_{n}\right)=\left\{\vee_{j \leq m} b_{j}: m \in \mathbb{N}\right\} .
$$

As before, $G\left(T_{1}, \ldots, T_{n}\right)$ is an element of $\mathcal{A}$.

If $\sigma$ is a winning strategy for ONE, then by [H7] and [H8] also $G$ is a winning strategy for ONE.

We now show that for Hurewicz pairs $(\mathcal{A}, \mathcal{B})$ the selection principle $\mathrm{S}_{\text {fin }}(\mathcal{A}, \mathcal{B})$ is characterized by the game $\mathrm{G}_{f i n}(\mathcal{A}, \mathcal{B})$.

Theorem 7 (Fundamental Theorem for Hurewicz Pairs). If $(\mathcal{A}, \mathcal{B})$ is a Hurewicz pair, then the following are equivalent:

(1) $\mathrm{S}_{\text {fin }}(\mathcal{A}, \mathcal{B})$

(2) ONE has no winning strategy in $\mathrm{G}_{f i n}(\mathcal{A}, \mathcal{B})$.

Pr. 1 : Let $\left(A_{n}: n \in \mathbb{N}\right)$ be a sequence of elements of $\mathcal{A}$. Define for ONE the strategy $\sigma$ so that in the $n$-th inning $\sigma$ calls on ONE to play $A_{n}$. By 2 this is not a winning strategy for ONE. Consider a $\sigma$-play lost by ONE, say

$$
A_{1}, T_{1}, \ldots, A_{n}, T_{n}, \ldots
$$

where for each $n$ the set $T_{n}$ is a finite subset of $A_{n}$. Since ONE loses this play we have $\cup_{n \in \mathbb{N}} T_{n} \in \mathcal{B}$.

$1 \Rightarrow 2$ : Let $\sigma$ be a strategy for ONE. By Lemmas 5 and 6 we may assume that $\sigma$ has the properties that 
- In each inning, $\sigma$ calls on ONE to play an $\omega$-chain which is a member of $\mathcal{A}$ and

- For each finite sequence $\left(T_{1}, \ldots, T_{n}\right)$ of finite sets such that $T_{1} \subseteq \sigma(\emptyset)$ and for $1<j \leq n T_{j} \subset \sigma\left(T_{1}, \ldots, T_{j-1}\right)$, we have $\vee T_{n} \leq C$ for each $C \in \sigma\left(T_{1}, \ldots, T_{n}\right)$.

Define the following array:

(1) $\left(T_{n}: n \in \mathbb{N}\right)$ enumerates $\sigma(\emptyset)$ in such a way that $m<n \Rightarrow T_{m}<T_{n}$

(2) With $T_{i_{1}, \ldots, i_{n}}$ defined for each $\left(i_{1}, \ldots, i_{n}\right) \in{ }^{n} \mathbb{N}$, let $\left(T_{i_{1}, \ldots, i_{n}, k}: k \in \mathbb{N}\right)$ enumerate $\sigma\left(T_{i_{1}}, T_{i_{1}, i_{2}}, \ldots, T_{i_{1}, \ldots, i_{n}}\right)$ in such a way that for $j<k$ we have $T_{i_{1}, \ldots, i_{n}, j}<T_{i_{1}, \ldots, i_{n}, k}$.

Then $\left(T_{\tau}: \tau \in<\omega \mathbb{N}\right)$ is, by the rules of the game $\mathrm{G}_{f i n}(\mathcal{A}, \mathcal{B})$, a Hurewicz $\mathcal{A}$-tree. Since $(\mathcal{A}, \mathcal{B})$ is a Hurewicz pair, and since $\mathrm{S}_{\text {fin }}(\mathcal{A}, \mathcal{B})$ holds, Theorem 2 implies that this tree has a $\mathcal{B}$-branch. Let $f \in{ }^{\omega} \mathbb{N}$ be given such that $\left(T_{f\lceil n}: n \in \mathbb{N}\right)$ is a $\mathcal{B}$-branch. Then

$$
\sigma(\emptyset), T_{f(1)}, \sigma\left(T_{f(1)}\right), T_{f(1), f(2)}, \sigma\left(T_{f(1)}, T_{f(1), f(2)}\right), \ldots
$$

is a $\sigma$-play of $\mathrm{G}_{f i n}(\mathcal{A}, \mathcal{B})$, and is lost by ONE.

For our applications below we will also need the following result:

Theorem 8. Let $(\mathcal{A}, \mathcal{B})$ be a Hurewicz family. Then the following are equivalent:

(1) $\mathrm{S}_{\text {fin }}(\mathcal{A}, \mathcal{B})$ holds.

(2) $\mathrm{S}_{\text {fin }}\left(\mathcal{A}_{\Omega}, \mathcal{B}\right)$ holds.

Proof. $1 \Rightarrow 2$ holds because $\mathcal{A}_{\Omega} \subseteq \mathcal{A}$. To see that $2 \Rightarrow 1$ holds, let a sequence $\left(A_{n}\right.$ : $n=1,2,3, \ldots)$ of elements of $\mathcal{A}$ be given. For each $n$ define: $A_{n}^{*}=\left\{\vee F: F \subset A_{n}\right\}$ By 3 of Lemma 3 , each $A_{n}^{*}$ is in $\mathcal{A}_{\Omega}$. Apply $\mathrm{S}_{\text {fin }}\left(\mathcal{A}_{\Omega}, \mathcal{B}\right)$ to $\left(A_{n}^{*}: n=1,2,3, \ldots\right)$ : For each $n$ fix a finite set $F_{n} \subset A_{n}^{*}$ such that $\mathbf{B}=\cup_{n<\infty} F_{n}$ is in $\mathcal{B}$. For each $n$, and for each $x \in F_{n}$, choose a finite subset $G_{x}$ of $A_{n}$ with $x=\vee G_{x}$. Put $V_{n}=\cup\left\{G_{x}: x \in F_{n}\right\}$, and finally put $V=\cup_{n<\infty} V_{n}$. Observe that for each $n, V_{n}$ is a finite subset of $A_{n}$. Since $V$ is a subset of $\cup \mathcal{A}$, and $\mathbf{V}=\{\vee F: F \subset V$ finite $\}$ is refined by the member $\mathbf{B}$ of $\mathcal{B},[\mathrm{H} 8]$ implies that $\mathbf{V}$ is an element of $\mathcal{B}$. But then [H7] implies that $V$ is an element of $\mathcal{B}$.

Applications. Let $(X, \tau)$ be a topological space and let $Y$ be a subset of $X$.

Let $\mathcal{O}_{X}$ denote the set of open covers of $X$, and let $\mathcal{O}_{X Y}$ denote the set of covers of $Y$ by sets open in $X$. If $X$ is a Lindelöf space then $\left(\mathcal{O}_{X}, \mathcal{O}_{X Y}\right)$ is a Hurewicz pair. In particular, $\mathcal{O}_{X}$ is a Hurewicz family. Thus $\mathrm{S}_{f i n}\left(\mathcal{O}_{X}, \mathcal{O}_{X Y}\right)$ holds if, and only if, ONE has no winning strategy in the game $\mathrm{G}_{f i n}\left(\mathcal{O}_{X}, \mathcal{O}_{X Y}\right)$. This result for the case $X=Y$ is Theorem 10 of [8]. The general case is Theorem 3.4.4 of [1].

Let $\mathcal{O}_{\Omega(X)}$ denote $\left(\mathcal{O}_{X}\right)_{\Omega}$ as per Definition 5 and let the symbol $\Omega_{X}$ denotes the collection of $\omega$-covers of $X$. An open cover $\mathcal{U}$ of $X$ is an $\omega$-cover if $X \notin$ $\mathcal{U}$, and if there is for each finite subset $F$ of $X$ a $U \in \mathcal{U}$ such that $F \subset U$. One can show that $\Omega_{X}$ consists of those members of $\mathcal{O}_{\Omega(X)}$ which do not have $X$ as a member. Theorem 8 implies that $\mathrm{S}_{f i n}\left(\mathcal{O}_{X}, \mathcal{O}_{X Y}\right)$ holds if, and only if, 
$\mathrm{S}_{f i n}\left(\Omega_{X}, \mathcal{O}_{X Y}\right)$ holds. Observe that since $\Omega_{X}$ is a subset of $\mathcal{O}_{X}$, it follows that if ONE has no winning strategy in $\mathrm{G}_{f i n}\left(\mathcal{O}_{X}, \mathcal{O}_{X Y}\right)$, then also ONE has no winning strategy in the game $\mathrm{G}_{f i n}\left(\Omega_{X}, \mathcal{O}_{X Y}\right)$, because in the latter game ONE is even more restricted in possible moves. This in turn implies that the selection hypothesis $\mathrm{S}_{f i n}\left(\Omega_{X}, \mathcal{O}_{X Y}\right)$ holds. Thus we obtain:

Corollary 9. If $(X, \tau)$ is a Lindelöf space and $Y$ is a subspace of $X$, the following are equivalent:

(1) $\mathrm{S}_{\text {fin }}\left(\mathcal{O}_{X}, \mathcal{O}_{X Y}\right)$ holds;

(2) ONE has no winning strategy in the game $\mathrm{G}_{\text {fin }}\left(\mathcal{O}_{X}, \mathcal{O}_{X Y}\right)$;

(3) ONE has no winning strategy in the game $\mathrm{G}_{\text {fin }}\left(\Omega_{X}, \mathcal{O}_{X Y}\right)$;

(4) $\mathrm{S}_{\text {fin }}\left(\Omega_{X}, \mathcal{O}_{X Y}\right)$ holds.

The hard implication here was that $1 \Rightarrow 2$.

Let $\mathcal{D}_{X}$ be the collection of families $\mathcal{U}$ of open sets with $\cup \mathcal{U}$ dense in $X$. Let $\mathcal{D}_{\Omega(X)}$ denote $\left(\mathcal{D}_{X}\right)_{\Omega}$ as per Definition 5. Extending the notation of [14], let $\Omega_{\mathcal{D}_{X}}$ denote the collection of elements $\mathcal{U}$ of $\mathcal{D}_{X}$ such that no element of $\mathcal{U}$ is a dense subset of $X$ and for each finite set $\mathcal{F}$ of nonempty open subsets of the space there is an $A \in \mathcal{U}$ such that for each $F \in \mathcal{F}, F \cap A \neq \emptyset$. One can show that $\Omega_{\mathcal{D}_{X}}$ consists of those members of $\mathcal{D}_{\Omega(X)}$ which do not have dense subsets of $X$ as members. For the subspace $Y$ of $X$ let $\mathcal{D}_{X Y}$ denote the set of the families $\mathcal{U}$ of open subsets of $X$ for which $(\cup \mathcal{U}) \cap Y$ is a dense subset of $Y$.

Then $\left(\mathcal{D}_{X}, \mathcal{D}_{X Y}\right)$ is a Hurewicz pair. In particular, $\mathcal{D}_{X}$ is a Hurewicz family. Thus, $\mathrm{S}_{\text {fin }}\left(\mathcal{D}_{X}, \mathcal{D}_{X Y}\right)$ holds if, and only if, ONE has no winning strategy in the game $\mathrm{G}_{f i n}\left(\mathcal{D}_{X}, \mathcal{D}_{X Y}\right)$. The version where $Y=X$ of this result is Theorem 2 of [14].

Since $\Omega_{\mathcal{D}_{X}}$ is a subset of $\mathcal{D}_{X}$, it follows that if ONE has no winning strategy in $\mathrm{G}_{\text {fin }}\left(\mathcal{D}_{X}, \mathcal{D}_{X Y}\right)$, then ONE has no winning strategy in $\mathrm{G}_{\text {fin }}\left(\Omega_{\mathcal{D}_{X}}, \mathcal{D}_{X Y}\right)$. This in turn implies in an elementary way that $S_{f i n}\left(\Omega_{\mathcal{D}_{X}}, \mathcal{D}_{X Y}\right)$ holds. Theorem 8 implies that $\mathrm{S}_{f i n}\left(\mathcal{D}_{\Omega(X)}, \mathcal{D}_{X Y}\right)$ holds if, and only if, $\mathrm{S}_{f i n}\left(\mathcal{D}_{X}, \mathcal{D}_{X Y}\right)$ holds. And one can also show that $\mathrm{S}_{f i n}\left(\Omega_{\mathcal{D}_{X}}, \mathcal{D}_{X Y}\right)$ is equivalent to $\mathrm{S}_{f i n}\left(\mathcal{D}_{\Omega(X)}, \mathcal{D}_{X Y}\right)$. Thus we obtain the following generalization of Theorem 4 of [14]:

Corollary 10. If $(X, \tau)$ is a Lindelöf space and $Y$ is a subspace of $X$, the following are equivalent:

(1) $\mathrm{S}_{\text {fin }}\left(\mathcal{D}_{X}, \mathcal{D}_{X Y}\right)$ holds;

(2) ONE has no winning strategy in the game $\mathrm{G}_{\text {fin }}\left(\mathcal{D}_{X}, \mathcal{D}_{X Y}\right)$;

(3) ONE has no winning strategy in the game $\mathrm{G}_{\text {fin }}\left(\Omega_{\mathcal{D}_{X}}, \mathcal{D}_{X Y}\right)$;

(4) $\mathrm{S}_{\text {fin }}\left(\Omega_{\mathcal{D}_{X}}, \mathcal{D}_{X Y}\right)$ holds.

Again, the hard implication is $1 \Rightarrow 2$.

As a further application we obtain the following result on Pixley-Roy duality: For space $X$ and subspace $Y$ of $X$, let $\operatorname{PR}(X)$ and $\operatorname{PR}(Y)$ respectively denote the Pixley-Roy hyperspaces of $X$ and $Y$. See for example [16] for an elementary introduction. Above we introduced already $\Omega_{X}$. The symbol $\Omega_{X Y}$ denotes those 
families $\mathcal{U}$ of open subsets of $X$ with the property that there is for each finite subset $F$ of $Y$ an element $U \in \mathcal{U}$ with $F \subset U$. In Theorem 3.3.6 of [1] it was shown that the following two statements are equivalent:

(1) For each $n, \mathrm{~S}_{f i n}\left(\mathcal{O}_{X^{n}}, \mathcal{O}_{X^{n} Y^{n}}\right)$ holds;

(2) $\mathrm{S}_{f i n}\left(\Omega_{X}, \Omega_{X Y}\right)$ holds.

Using the techniques in the proof of Theorem 6 of [16] one obtains the following generalization of corresponding results of [5] and [14]:

Corollary 11. Let $X$ be a separable metric space and let $Y$ be a subspace of $X$. The following are equivalent:

(1) $\mathrm{S}_{\text {fin }}\left(\mathcal{D}_{\mathrm{PR}(X)}, \mathcal{D}_{\mathrm{PR}(X) \mathrm{PR}(Y)}\right)$ holds;

(2) For each $n, \mathrm{~S}_{\text {fin }}\left(\mathcal{O}_{X^{n}}, \mathcal{O}_{X^{n} Y^{n}}\right)$ holds;

(3) $\mathrm{S}_{\text {fin }}\left(\Omega_{X}, \Omega_{X Y}\right)$ holds.

\section{Ramsey families: From $\mathrm{G}_{f i n}(\mathcal{A}, \mathcal{B})$ to $\mathcal{A} \rightarrow\lceil\mathcal{B}\rceil_{k}^{2}$.}

In this section we show how the game $\mathrm{G}_{f i n}(\mathcal{A}, \mathcal{B})$ can be used to verify that the partition relation $\mathcal{A} \rightarrow\lceil\mathcal{B}\rceil_{k}^{2}$ is true in when $\mathcal{A}$ has some special properties.

Definition 6. A family $\mathcal{A}$ of subsets of a lattice $\mathbb{L}$ is said to be a Ramsey family if for each $A \in \mathcal{A}$ and each $k$, for each partition $A=\cup_{j \leq k} A_{j}$ of $A$ into $k$ pieces, there is a $j \leq k$ such that $A_{j} \in \mathcal{A}$.

Thus, in our earlier notation of Definition $5, \mathcal{A}_{\Omega}$ is a Ramsey family.

Theorem 12 (Fundamental Theorem of Ramsey Families). Let $\mathcal{A}$ and $\mathcal{B}$ be families of subsets of the lattice $\mathbb{L}$ such that $\mathcal{A}$ is Lindelöf. If $\mathcal{A}$ is a Ramsey family and if ONE has no winning strategy in the game $\mathrm{G}_{\text {fin }}(\mathcal{A}, \mathcal{B})$, then for each $k \in \mathbb{N}$ the partition relation $\mathcal{A} \rightarrow\lceil\mathcal{B}\rceil_{k}^{2}$ holds.

Proof. Let $A \in \mathcal{A}$ as well as a positive integer $k$ be given. We may assume that $A$ is countable. Enumerate $A$ bijectively as $\left(a_{n}: n<\infty\right)$. Fix a function $f:[A]^{2} \rightarrow\{1, \ldots, k\}$. Recursively define a sequence $\left(A_{n}: n<\infty\right)$ of subsets of $A$, and a sequence $\left(i_{n}: n<\infty\right)$ of elements of $\{1, \ldots, k\}$ so that:

(1) For each $n, A_{n} \in \mathcal{A}$ and $A_{n+1} \subset A_{n}$;

(2) For each $n, A_{n+1}=\left\{a_{j} \in A_{n}: j>n+1\right.$ and $\left.f\left(\left\{a_{n+1}, a_{j}\right\}\right)=i_{n+1}\right\}$.

To see that this can be done, first observe that putting $B_{j}=\left\{a_{i} \in A: i>\right.$ 1 and $\left.f\left(\left\{a_{1}, a_{i}\right\}\right)=j\right\}$ we get a partition of $A \backslash\left\{a_{1}\right\}$ into finitely many pieces. Since $\mathcal{A}$ is a Ramsey family there is a $j$ for which $B_{j}$ is in $\mathcal{A}$. Fix such a $j$ and set $i_{1}=j, A_{1}=B_{j}$. Next observe that by similar argument the remaining terms of an infinite sequence as above can be selected consecutively.

Next, for each $j \in\{1, \ldots, k\}$ define $E_{j}=\left\{a_{n}: i_{n}=j\right\}$. For each $n, A_{n} \cap E_{1}$, $\ldots, A_{n} \cap E_{k}$ partitions $A_{n}$ into finitely many pieces, and so there is a $j_{n}$ with $A_{n} \cap E_{j_{n}}$ in $\mathcal{A}$. Since for each $n$ we have $A_{n} \supset A_{n+1}$, we may assume that the same $j$ works for all $A_{n}$ 's. Fix such a $j$. 
Now define the following strategy, $\sigma$, for ONE in the game $\mathrm{G}_{\text {fin }}(\mathcal{A}, \mathcal{B})$ : In the first inning, $\sigma(\emptyset)=A_{1} \cap E_{j}$. If TWO responds by choosing a finite set $T_{1} \subset \sigma(\emptyset)$, let $n_{1}$ be $1+\max \left\{n: a_{n} \in T_{1}\right\}$. Then ONE plays $\sigma\left(T_{1}\right)=A_{n_{1}} \cap E_{j}$. If TWO responds with finite set $T_{2} \subset \sigma\left(T_{1}\right)$, and put $n_{2}=1+\max \left\{n: a_{n} \in T_{2}\right\}$. Then ONE plays $\sigma\left(T_{1}, T_{2}\right)=A_{n_{2}} \cap E_{j}$, and so on. (Observe that $n_{1}<n_{2}$.)

Since ONE has no winning strategy in $\mathrm{G}_{\text {fin }}(\mathcal{A}, \mathcal{B})$, there is a play during which ONE used $\sigma$ but lost. Let

$$
\sigma(\emptyset), T_{1}, \sigma\left(T_{1}\right), T_{2}, \sigma\left(T_{1}, T_{2}\right), T_{3}, \ldots
$$

be such a play lost by ONE. Then as TWO won we have $B=\cup_{n \in \mathbb{N}} T_{n} \in \mathcal{B}$. By the definition of the strategy $\sigma$ we also have for $r \neq s$ that $n_{r} \neq n_{s}$, and $f(\{a, b\})=j$ whenever $a$ and $b$ are from distinct $T_{j}$ 's.

Applications. There are many examples of Ramsey families in the literature. We give some applications of Theorem 12 to these.

With $(X, \tau)$ a Lindelöf topological space and let $Y$ be a subset of $X,\left(\mathcal{O}_{X}, \mathcal{O}_{Y}\right)$ is a Hurewicz pair and $\mathcal{O}_{X}$ is a Hurewicz family. Moreover, $\Omega_{X}$ is a Ramsey family. According to Gerlits and Nagy (see [7]) a space is said to be an $\epsilon$-space if $\Omega_{X}$ is a Lindelöf family.

Corollary 13. If $(X, \tau)$ is an $\epsilon$-space and $Y$ is a subspace of $X$, then $\mathrm{S}_{\text {fin }}\left(\mathcal{O}_{X}, \mathcal{O}_{Y}\right)$ implies that $\Omega_{X} \rightarrow\left\lceil\mathcal{O}_{Y}\right\rceil_{k}^{2}$ holds for each $k$.

Proof. We saw in Corollary 9 that $\mathrm{S}_{\text {fin }}\left(\mathcal{O}_{X}, \mathcal{O}_{Y}\right)$ is equivalent to ONE not having a winning strategy in $\mathrm{G}_{f i n}\left(\Omega_{X}, \mathcal{O}_{Y}\right)$. Apply Theorem 12.

The case when $X=Y$ of this corollary was obtained in Theorem 6 of [17].

It was noted in [14] (p. 21) that if each finite power of a space has countable cellularity then for that space the family $\mathcal{D}_{\Omega}$ is a Lindelöf family. In particular, separable metric spaces have this property. The case $X=Y$ of the following corollary was obtained in Theorem 10 of [14]:

Corollary 14. Let $(X, \tau)$ is a Lindelöf space such that $\mathcal{D}_{\Omega(X)}$ is a Lindelöf family. Let $Y$ be a subspace of $X$. If $\mathrm{S}_{\text {fin }}\left(\mathcal{D}_{X}, \mathcal{D}_{Y}\right)$ holds then $\mathcal{D}_{\Omega(X)} \rightarrow\left\lceil\mathcal{D}_{Y}\right\rceil_{k}^{2}$ holds for each $k$.

Proof. By Corollary $10 \mathrm{~S}_{\text {fin }}\left(\mathcal{D}_{X}, \mathcal{D}_{Y}\right)$ implies that ONE has no winning strategy in $\mathrm{G}_{f i n}\left(\mathcal{D}_{\Omega(X)}, \mathcal{D}_{Y}\right)$. Apply Theorem 12 .

And this in turn immediately gives the following generalization of (11) of Corollary 11 of [14]:

Corollary 15. Let $X$ be a separable metric space and let $Y$ be a subspace of $X$. If $\mathrm{S}_{\text {fin }}\left(\mathcal{D}_{\mathrm{PR}(X)}, \mathcal{D}_{\mathrm{PR}(Y)}\right)$ holds then for each $\left.k, \mathcal{D}_{\Omega(\mathrm{PR}(X))} \rightarrow\left\lceil\mathcal{D}_{\mathrm{PR}(Y)}\right)\right\rceil_{k}^{2}$ holds. 


\section{Selectable pairs: From $\mathcal{A} \rightarrow\lceil\mathcal{B}\rceil_{k}^{2}$ TO $\mathrm{S}_{\text {fin }}(\mathcal{A}, \mathcal{B})$.}

In this section we show how the partition relation $\mathcal{A} \rightarrow\lceil\mathcal{B}\rceil_{k}^{2}$ implies $\mathrm{S}_{\text {fin }}(\mathcal{A}, \mathcal{B})$ appropriate $\mathcal{A}$ and $\mathcal{B}$. We require the following notion: An element $A$ of $\mathcal{A}$ is said to be large if it is nonempty and for each finite subset $F$ of $\mathbb{L}, A \backslash F$ is in $\mathcal{A}$. The symbol $\mathcal{A}_{\Lambda}$ denotes the set $\{A \in \mathcal{A}: A$ is large $\}$.

Definition 7. $(\mathcal{A}, \mathcal{B})$ is a selectable pair if

$\mathrm{S} 1 \mathcal{A}$ is Lindelöf;

S2 The union of countably many members of $\mathcal{A}$ is a member of $\mathcal{A}$;

S3 If $A$ is a countable element of $\mathcal{A}, f: A \rightarrow \mathbb{N}$ is a function and $\left(B_{n}: n \in \mathbb{N}\right)$ is a sequence of elements of $\mathcal{A}$, then $\left\{a \wedge b: a \in A\right.$ and $\left.b \in B_{f(a)}\right\}$ is an element of $\mathcal{A}$;

S4 For each $a \in \cup \mathcal{A}, \mathcal{P}(\{b \in \mathbb{L}: b \leq a\}) \cap \mathcal{B}=\emptyset$;

$\mathrm{S} 5 \mathcal{B}=\mathcal{B}_{\Lambda}$

S6 If $C$ is a countable subset of $\cup \mathcal{A}$ such that there is a $B \in \mathcal{B}$ with $\{b \in B$ : $(\exists x \in C)(b \leq x)\} \in \mathcal{B}$, then $C$ is a member of $\mathcal{B}$.

Certain Hurewicz pairs are selectable pairs.

Lemma 16. If $(\mathcal{A}, \mathcal{B})$ is a Hurewicz pair, then it also has properties $\mathrm{S1}$, S2 and S6.

S1. is [H1]. [H3] implies [S2] as follows: Let $\left(A_{n}: n \in \mathbb{N}\right)$ be a sequence of elements of $\mathcal{A}$ and put $A=A 1$ and $B=\cup_{n<\infty} A_{n}$. Apply [H3].

[S6] follows from [H8] as follows: Let $C$ be a countable subset of $\mathbb{L}$ and let $B \in \mathcal{B}$ be such that $A:=\{b \in B:(\exists x \in C)(b \leq x)\}$ is in $\mathcal{B}$. Then $A$ refines $C$, and now apply [H8].

Theorem 17 (Fundamental Theorem of Selectable Pairs). If $(\mathcal{A}, \mathcal{B})$ is a selectable pair and $\mathcal{A} \rightarrow\lceil\mathcal{B}\rceil_{2}^{2}$, then $\mathrm{S}_{\text {fin }}(\mathcal{A}, \mathcal{B})$ holds.

Proof. Let $\left(\mathcal{U}_{n}: n<\infty\right)$ be a sequence of elements of $\mathcal{A}$. By [S1] we may assume each $\mathcal{U}_{n}$ is countable, and enumerate it as $\left(u_{k}^{n}: k<\infty\right)$. Define $\mathcal{V}$ to be the collection of elements of $\mathbb{L}$ of the form $u_{n}^{1} \wedge u_{k}^{n}$. By [S3] $\mathcal{V}$ is an element of $\mathcal{A}$. Choose for each element of $\mathcal{V}$ a representation of the form $u_{n}^{1} \wedge u_{k}^{n}$.

Define a function $f:[\mathcal{V}]^{2} \rightarrow\{1,2\}$ by

$$
f\left(\left\{u_{n_{1}}^{1} \wedge u_{k}^{n_{1}}, u_{n_{2}}^{1} \wedge u_{j}^{n_{2}}\right\}\right)= \begin{cases}1 & \text { if } n_{1}=n_{2} \\ 2 & \text { otherwise }\end{cases}
$$

Choose a nearly homogeneous of color $j \mathcal{W} \subseteq \mathcal{V}$ with $\mathcal{W} \in \mathcal{B}$. Let $\left(\mathcal{W}_{k}: k<\infty\right)$ be a sequence of finite sets, disjoint from each other and with union $\mathcal{W}$, such that for $a$ and $b$ from distinct $\mathcal{W}_{k}$ 's, $f(\{a, b\})=j$.

Case 1: $j=1$. Then there is an $n$ such that for all $a \in \mathcal{W}$ we have $a \leq u_{n}^{1}$. Since [S4] then implies that $\mathcal{W}$ is not an element of $\mathcal{B}$, Case 1 does not hold.

Case $2: j=2$. For each $k>1$ define $\mathcal{G}_{k}$ to be the set of $u_{j}^{k}$ which occur as second coordinate in the chosen representations of elements of $\mathcal{W}$. Then each $\mathcal{G}_{k}$ is a finite subset of $\mathcal{U}_{k}$. Put $\mathcal{G}=\bigcup_{k<\infty} \mathcal{G}_{k}$. 
We have $\mathcal{W}=\{b \in \mathcal{W}:(\exists x \in \mathcal{G})(b \leq x)\}$ is in $\mathcal{B}$, and each element of $\mathcal{G}$ is an element of some member of $\mathcal{A}$. Thus by [S6], $\mathcal{G}$ is an element of $\mathcal{B}$. By letting $\mathcal{G}_{k}$ be empty for those $k$ for which no $\mathcal{G}_{k}$ was defined, we find that the sequence $\left(\mathcal{G}_{k}: k<\infty\right)$ witnesses $\mathrm{S}_{\text {fin }}(\mathcal{A}, \mathcal{B})$ for $\left(\mathcal{U}_{k}: k<\infty\right)$.

\section{Applications}

Let $X$ be a space and $Y$ a subspace of $X$. Let $\Omega_{X Y}$ be the collection of $\omega$ covers $\mathcal{U}$ of $X$ such that no element of $\mathcal{U}$ covers $Y$ (so, $Y$ is infinite). Let $\Lambda_{Y}$ be the collection of large covers of $Y$ by sets open in $X$. If $X$ is an $\epsilon$-space, then $\left(\Omega_{X Y}, \Lambda_{Y}\right)$ is a Hurewicz pair. Indeed, $\left(\Omega_{X Y}, \Lambda_{Y}\right)$ is a selectable pair. Thus we obtain:

Corollary 18. If $X$ is an $\epsilon$-space then $\Omega_{X Y} \rightarrow\left\lceil\Lambda_{Y}\right\rceil_{2}^{2}$ implies that $\mathrm{S}_{f i n}\left(\Omega_{X Y}, \Lambda_{Y}\right)$ holds.

If for a space $X$ the family $\mathcal{D}_{\Omega(X)}$ is a Lindelöf family then the pair $\left(\mathcal{D}_{\Omega(X)}, \mathcal{D}_{X}\right)$ is a selectable pair. Thus we obtain Theoem 10 of [14]:

Corollary 19. If $\mathcal{D}_{\Omega(X)}$ is a Lindelöf family then the following are equivalent:

(1) $\mathcal{D}_{\Omega(X)} \rightarrow\left\lceil\mathcal{D}_{X}\right\rceil_{2}^{2}$;

(2) $\mathrm{S}_{\text {fin }}\left(\mathcal{D}_{X}, \mathcal{D}_{X}\right)$ holds.

For a space non-compact $X, \mathcal{K}$ denotes the compact-covering open covers of $X$, also said to be the $k$-covers of $X$ in [6]. An open cover $\mathcal{U}$ of $X$ is a $k$ cover if there is for every compact subset $K$ of $X$ an element $U$ of $\mathcal{U}$ such that $K \subseteq U$, and $X \notin$ mathcalU. $(\mathcal{K}, \mathcal{K})$ is a selectable pair, and thus we have the implication $(2) \Rightarrow(1)$ in Theorem 7 of $[6]$ :

Corollary 20. Let $X$ is a non-compact space for which $\mathcal{K}$ is a Lindelöf family. If $\mathcal{K} \rightarrow\lceil\mathcal{K}\rceil_{k}^{2}$ for some $k \geq 2$, then $\mathrm{S}_{\text {fin }}(\mathcal{K}, \mathcal{K})$ holds.

\section{REFERENCES}

[1] L. Babinkostova, Selektivni principi vo Topologijata, Ph.D. thesis, Sts Cyril and Methodius University, Skopje, Macedonia (2001).

[2] L. Babinkostova, Lj.D.R. Kočinac and M. Scheepers, Combinatorics of open covers (VIII), Topology and its Applications, 140/1 (2004), 15-32.

[3] M. Scheepers, $\mathrm{S}_{1}(\mathcal{A}, \mathcal{B})$ in distributive lattices, In: Selection Principles and Covering Properties in Topology (Lj.D.R. Kočinac, ed.), Quaderni di Matematica, Vol. 18, Caserta, 2006.

[4] J.E. Baumgartner and A.D. Taylor, Partition theorems and ultrafilters, Transactions of the American Mathematical Society, 241 (1978), 283-309.

[5] P. Daniels, Pixley-Roy spaces over subsets of the reals, Topology and its Applications, 29 (1988), 93-106.

[6] G. Di Maio, Lj.D.R. Kočinac, E. Meccariello, Applications of k-covers, Acta Mathematica Sinica English Series, 22:4 (2006), 1151-1160. 
[7] J. Gerlits and Zs. Nagy, Some properties of $C(X)$, I, Topology and its Applications, 14 (1982), 151-161.

[8] W. Hurewicz, Über eine Verallgemeinerung des Borelschen Theorems, Mathematische Zeitschrift, 24 (1925), 401-425.

[9] W. Just, A.W. Miller, M. Scheepers and P.J. Szeptycki, Combinatorics of open covers (II), Topology and its Applications, 73 (1996), 241-266.

[10] Lj.D.R. Kočinac and M. Scheepers, Combinatorics of open covers (VII): groupability, Fundamenta Mathematicae, 179 (2003), 131-154.

[11] M. Scheepers, Combinatorics of Open covers (I): Ramsey Theory, Topology and its Applications, 69 (1996), 31-62.

[12] M. Scheepers, Combinatorics of open covers (III): Games, $C_{p}(X)$, Fundamenta Mathematicae, 12 (1997), 231-254.

[13] M. Scheepers, Combinatorics of open covers (IV): Subspaces of the Alexandroff double of the unit interval, Topology and its Applications, 83 (1998), 63-75.

[14] M. Scheepers, Combinatorics of open covers (V): Pixley-Roy spaces of sets of reals, and $\omega$-covers, Topology and its Applications, 102 (2000), 13-31.

[15] M. Scheepers, Combinatorics of open covers (VI): Quaestiones Mathematicae, 22 (1999), $109-130$.

[16] M. Scheepers, The relative Rothberger property and Pixley-Roy spaces, Mathematica Macedonica, 1 (2003), 15-19.

[17] M. Scheepers, Open covers and partition relations, Proceedings of the American Mathematical Society, 127 (1999), 577-581.

\author{
Department of Mathematics \\ Boise State University \\ BOISE \\ IDAHO 83725 \\ USA \\ E-mail address: marion@diamond.boisestate.edu
}

